\title{
Numerical Model on Sound-Solid Coupling in Human Ear and Study on Sound Pressure of Tympanic Membrane
}

\author{
Yao Wen-juan, ${ }^{1,2}$ Ma Jian-wei, ${ }^{1}$ and Hu Bao-linn ${ }^{1,2}$ \\ ${ }^{1}$ Department of Civil Engineering, P.O. Box 47, No. 149 Yanchang Road, Shanghai University, \\ Shanghai 200072, China \\ 2 Shanghai Institute of Applied Mathematics and Mechanics, Shanghai 200072, China \\ Correspondence should be addressed to Yao Wen-juan, wjyao@shu.edu.cn
}

Received 28 March 2011; Accepted 8 August 2011

Academic Editor: Alexei Mailybaev

Copyright (C) 2011 Yao Wen-juan et al. This is an open access article distributed under the Creative Commons Attribution License, which permits unrestricted use, distribution, and reproduction in any medium, provided the original work is properly cited.

\begin{abstract}
Establishment of three-dimensional finite-element model of the whole auditory system includes external ear, middle ear, and inner ear. The sound-solid-liquid coupling frequency response analysis of the model was carried out. The correctness of the FE model was verified by comparing the vibration modes of tympanic membrane and stapes footplate with the experimental data. According to calculation results of the model, we make use of the least squares method to fit out the distribution of sound pressure of external auditory canal and obtain the sound pressure function on the tympanic membrane which varies with frequency. Using the sound pressure function, the pressure distribution on the tympanic membrane can be directly derived from the sound pressure at the external auditory canal opening. The sound pressure function can make the boundary conditions of the middle ear structure more accurate in the mechanical research and improve the previous boundary treatment which only applied uniform pressure acting to the tympanic membrane.
\end{abstract}

\section{Introduction}

With the development of interdiscipline, the research that explores issues of life sciences with principles of mechanics has become a new frontier. The study of ear biological mechanics has a relatively brief history which trace back to the end of last century and the beginning of this century. Scholars mainly adopt two methods to study ear problems with mechanics: the first one is theoretical research methods, such as the use of analytical solution to eardrum vibration problem deduced by mechanical theory and analytical method of artificial ossicle detection [1, 2]; the other is numerical modeling methods, the most popular of which is 
finite element method. For example, Voss and Peake [3] studied the relationship between sound transmission and perforation using the finite element method. Bance et al. [4] studied the impact of size of incus prosthesis head on Tympanic membrane vibration. Dai et al. [5] studied the combined effects of fluid and air in middle ear cavity. Vard and Kelly [6] studied how the design of ventilation tubes influence on vibration. Tange and Grolman [7] studied the impact of connector shape of stapes replacement prosthesis on hearing conduction. Tenney et al. [8] studied the restoration of hearing of stapes prosthesis in the short term and the impact of angle of implantation on hearing. Gan et al. [9-11] simulated the tympanic membrane perforation, inner ear impedance, and other middle ear diseases using the finite element method. Wenjuan Yao and coworkers used finite element methods to analyse the material of the stapes replacement prosthesis and the connection between prosthesis and incus long legs [12-14]. The above studies have promoted the development of earbiomechanics.

However, these preliminary studies all simplified the boundary conditions, in which the sound incentive on the tympanic membrane surface was defined as uniformly distributed loads. The external load on the tympanic membrane, however, is not really uniform, because sound waves have reached external auditory canal before they reach tympanic membrane, and gas-solid coupling occurs in external auditory canal then reach tympanic membrane, and fluid-solid coupling will happen between tympanic membrane and air in the external auditory canal. After sound-solid-liquid three-phase fluid-solid coupling occurs, the pressure distribution on the tympanic membrane is shown in Figure 1.

\section{Model and Method}

\subsection{Data Sources and Establishment of Middle Ear Model}

Based on the CT scan images from Zhongshan Hospital of Fudan University on the normal human middle ear (GE lights peed VCT 64 Slice spiral CT machine, Scanning parameters: collimation $0.625 \mathrm{~mm}$, tube rotation time $0.4 \mathrm{~s}$, reconstruction thickness $0.625 \mathrm{~mm}$, interval $0.5 \sim 0.625 \mathrm{~mm}$.) by further processing the image, using self-compiled program to Numerical Value the CT scans and import it into FE software Patran to reconstruct three-dimensional finite element model of ear structure, then divide into grid, we can define the boundary conditions and the material parameters, as shown in Figures 2 and 3. The model adopts interface element to simulate interosseous membrane among ossicles in order that transfer behavior among ossicles (malleus, incus, and stapes) is simulated perfectly.

This paper combined the numerical analysis and theoretical analysis to study the load distribution on the eardrum deeply.

External auditory canal gas unit is divided into 7200 eight-node hexahedron (Hex8) units. The number of nodes is 7460 . Tympanic membrane is divided into 330 four-node quadrilateral (Quad4) and 30 triangle (Tri3) surface units, the number of nodes is 373. Ossicular chain is divided into 21,438 four-node tetrahedral elements (Tet4), nodes 6065, Figures 2 and 3.

Cochlea mesh: the fluid domain near stapes within vestibular is divided into Tet4 units, and other fluid domain are divided into Hex8 units, The fluid unit attributes are defined as FLUID units, the number of units produced is 4391 in total, 6817 nodes; oval window is divided into Tria3 surface units, oval window unit is defined as two-dimensional membrane structure, and the number of units is 56 in total, 37 nodes; and round window is divided 


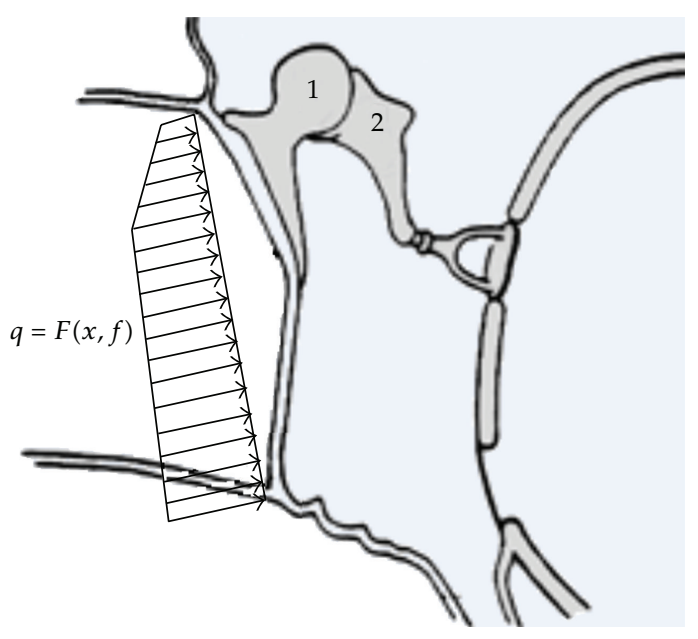

Figure 1: Load on the eardrum.

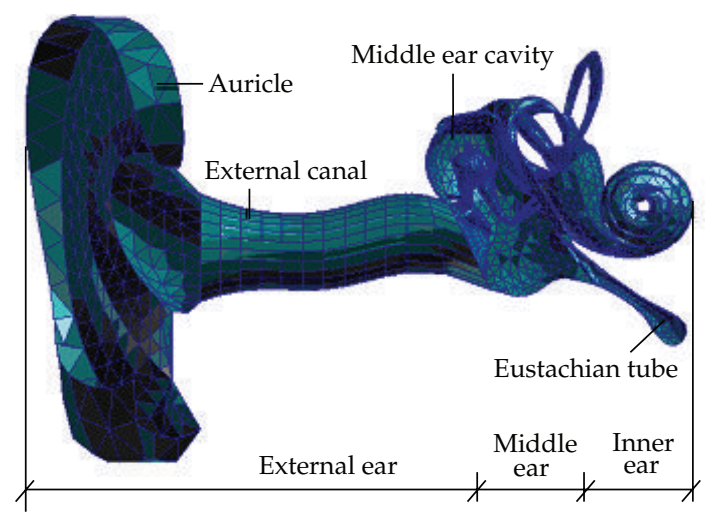

Figure 2: FE model of human ear.

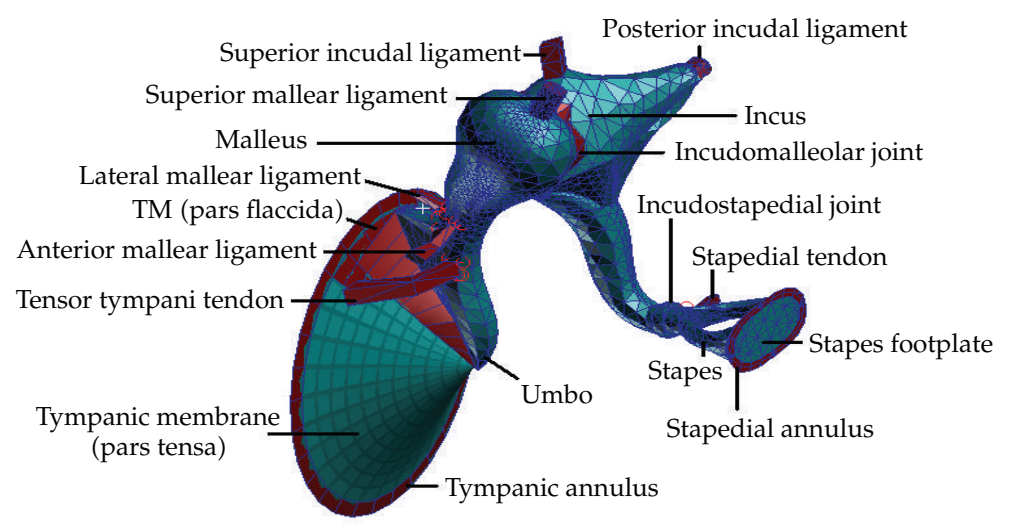

Figure 3: FE model of middle ear in detail. 
into Quad4 surface units, and round window membrane unit is defined as two-dimensional structure (membrane) total 16 units, 25 nodes. Mesh is shown in Figure 3.

\subsection{Governing Equations}

The structural dynamics equation of the acoustic structural coupled system of air in external canal and tympanic membrane, stapes footplate, and perilymphatic fluid in inner ear

$$
\left[\begin{array}{cc}
{\left[M_{e}\right]} & {[0]} \\
{\left[M^{f s}\right]} & {\left[M_{e}^{p}\right]}
\end{array}\right]\left\{\begin{array}{l}
\ddot{u}_{e} \\
\ddot{P}_{e}
\end{array}\right\}+\left[\begin{array}{cc}
{\left[C_{e}\right]} & {[0]} \\
{[0]} & {\left[C_{e}^{p}\right]}
\end{array}\right]\left\{\begin{array}{l}
\dot{u}_{e} \\
\dot{P}_{e}
\end{array}\right\}+\left[\begin{array}{cc}
{\left[K_{e}\right]} & {\left[K^{f s}\right]} \\
{[0]} & {\left[K_{e}^{p}\right]}
\end{array}\right]\left\{\begin{array}{l}
\left\{u_{e}\right\} \\
\left\{P_{e}\right\}
\end{array}\right\}=\left\{\begin{array}{c}
\left\{F_{e}\right\} \\
\{0\}
\end{array}\right\},
$$

where

$$
\begin{gathered}
{\left[M^{f s}\right]=\rho_{o}\left[R_{e}\right]^{T},} \\
{\left[K^{f_{s}}\right]=-\left[R_{e}\right],}
\end{gathered}
$$

$\left[M_{e}\right]$ is structural mass matrix; $\left[M^{f s}\right]$ is coupling interface mass matrix; $\left[M_{e}^{p}\right]$ is air mass matrix; $\left[C_{e}\right]$ is structural damping matrix; $\left[C_{e}^{p}\right]$ is air damping matrix; $\left[K_{e}^{p}\right]$ is fluid stiffness matrix; $u_{e}$ is the displacement vector; $P_{e}$ is the sound pressure matrix.

\subsection{Interface Fundamental Equations}

The assumption that elements are in no thickness

$$
\left\{\begin{array}{c}
\tau_{x} \\
\tau_{y} \\
\sigma_{n}
\end{array}\right\}=\left[\begin{array}{ccc}
K_{s} & 0 & 0 \\
0 & K_{s} & 0 \\
0 & 0 & K_{n}
\end{array}\right]\left\{\begin{array}{c}
\Delta u \\
\Delta v \\
\Delta w
\end{array}\right\}=[D]\left\{\begin{array}{c}
\Delta u \\
\Delta v \\
\Delta w
\end{array}\right\}
$$

where $K_{s}$ is tangential stiffness; $K_{n}$ is normal stiffness; $x, y$, and $n$ are two coordinates directions and element normal direction in the actual contact surface; $\Delta u, \Delta v$, and $\Delta w$ are relative displacements of upper wall and bottom wall of contact face in tangential and normal direction

$$
\left\{\begin{array}{c}
\Delta u \\
\Delta v \\
\Delta w
\end{array}\right\}=[B]\{\delta\}^{\ell}
$$


where

$$
\begin{gathered}
{[B]=\left[\begin{array}{cccccccccccccc}
-N_{1} & 0 & 0 & \cdots & -N_{4} & 0 & 0 & N_{1} & 0 & 0 & \cdots & N_{4} & 0 & 0 \\
0 & -N_{1} & 0 & \cdots & 0 & -N_{4} & 0 & 0 & N_{1} & 0 & \cdots & 0 & N_{4} & 0 \\
0 & 0 & -N_{1} & \cdots & 0 & 0 & -N_{4} & 0 & 0 & N_{1} & \cdots & 0 & 0 & N_{4}
\end{array}\right]} \\
\{\delta\}^{\ell}=\left[\begin{array}{lllllll}
u_{1} & v_{1} & w_{1} & \cdots & u_{8} & v_{8} & w_{8}
\end{array}\right] \\
N_{i}=\frac{1}{4}\left(1+\xi_{i} \zeta\right)\left(1+\eta_{i} \eta\right) \\
i=1,2,3,4 .
\end{gathered}
$$

Element stiffness matrix is

$$
\{R\}^{e}=\iint_{A}[B]^{T}[D][B] d v d y=\iint_{-1}^{1}[B]^{T}[D][B]|J| d \xi d \eta
$$

\subsection{Material Properties}

Material properties and acoustic properties of various parts of numerical models in this paper refer to experimental data in $[9,11,15]$, the relevant parameter values of them are shown in Tables 1 and 2, and Poisson's ratio was taken as 0.3 . Hearing system damping coefficient was taken as 0.5 by spreadsheet simulation.

\subsection{Boundary Conditions}

Because of the sensitivity of displacement of microstructure to the dynamical response of ear structure, the connection between soft tissue and temporal bone was regarded as fixed constraint, which is to say its displacement in all three orthogonal directions is zero. The defined boundary condition is listed below.

(1) $90 \mathrm{~dB}$ SPL (0.632 Pa, from $200 \mathrm{~Hz}$ to $8000 \mathrm{~Hz}$ ) was set on the opening surface of external auditory canal.

(2) The displacement of connection between soft tissues and temporal bone was defined to be zero in three orthogonal directions.

(3) The displacement of outer edge of tympanic membrane annular ligament was defined to be zero in three orthogonal directions.

(4) The displacement of outer edge of stapes annular ligament was defined to be zero in three orthogonal directions.

(5) The displacement of outer edge of oval window and round window was defined to be zero in three orthogonal directions.

(6) The displacement of external auditory canal wall and the inner ear bony labyrinth wall was defined to be zero in three orthogonal directions.

(7) Eardrum and Oval window are fluid-structure coupling interface. 
Table 1: Material properties of the FE model.

\begin{tabular}{lcc}
\hline Structure & Density $\left(\mathrm{kg} / \mathrm{m}^{3}\right)$ & Young's modulus $(\mathrm{Pa})$ \\
\hline Tympanic membrane (Pars tensa) & $1.2 \times 10^{3}$ & $3.5 \times 10^{7}$ \\
Tympanic membrane (Pars flaccida) & $1.2 \times 10^{3}$ & $2.0 \times 10^{7}$ \\
The tympanic membrane malleus attachment at the umbo & $1.2 \times 10^{3}$ & $3.5 \times 10^{7}[11]$ \\
The tympanic membrane malleus attachment at malleus handle & $1.2 \times 10^{3}$ & $3.5 \times 10^{3}[11]$ \\
Malleus head & $2.55 \times 10^{3}$ & $1.41 \times 10^{10}$ \\
Malleus neck & $4.53 \times 10^{3}$ & $1.41 \times 10^{10}$ \\
Malleus handle & $3.7 \times 10^{3}$ & $1.41 \times 10^{10}$ \\
Incudomalleolar joint & $3.2 \times 10^{3}$ & $1.41 \times 10^{10}$ \\
Incus body & $2.36 \times 10^{3}$ & $1.41 \times 10^{10}$ \\
Incus short process & $2.26 \times 10^{3}$ & $1.41 \times 10^{10}$ \\
Incus long process & $5.08 \times 10^{3}$ & $1.41 \times 10^{10}$ \\
Incudostapedial joint & $1.2 \times 10^{3}$ & $6.0 \times 10^{5}$ \\
Stapes & $2.2 \times 10^{3}$ & $1.41 \times 10^{10}$ \\
Superior mallear ligament & $2.5 \times 10^{3}$ & $4.9 \times 10^{6}$ \\
Lateral mallear ligament & $2.5 \times 10^{3}$ & $6.7 \times 10^{6}$ \\
Anterior mallear ligament & $2.5 \times 10^{3}$ & $2.1 \times 10^{7}$ \\
Superior incudal ligament & $2.5 \times 10^{3}$ & $4.9 \times 10^{4}$ \\
Posterior incudal ligament & $2.5 \times 10^{3}$ & $6.5 \times 10^{6}$ \\
Tensor tympani tendon & $2.5 \times 10^{3}$ & $8.7 \times 10^{6}$ \\
Stapedial tendon & $2.5 \times 10^{3}$ & $5.2 \times 10^{6}$ \\
Oval window & $1.2 \times 10^{3}$ & $5.5 \times 10^{6}$ \\
Round window membrane & $1.2 \times 10^{3}$ & $3.5 \times 10^{5}$ \\
Basilar membrane & $1.0 \times 10^{3}$ & $2.0 \times 10^{5}$ \\
\hline
\end{tabular}

Table 2: Acoustic properties of ear components.

\begin{tabular}{lcc}
\hline Structure & Density $\left(\mathrm{kg} / \mathrm{m}^{3}\right)$ & Speed $(\mathrm{m} / \mathrm{s})$ \\
\hline Air & 1.21 & 340 \\
Perilymphatic fluid & 1000 & 1400 \\
\hline
\end{tabular}

\section{Results}

\subsection{The Reliability of Numerical Simulations}

Figures 4 and 5 shows the FE model-derived frequency response curve of the TM displacement and stapes footplate displacement in comparison with the corresponding curves obtained from 10 temporal bones at the same input sound pressure level of $90 \mathrm{~dB}$ applied on the TM in the ear canal by Gan et al. [15]. These figures show that the FE model predicted TM and stapes footplate curves fall into the range of the 10 temporal bone experimental curves and the tendency is similar across the frequency range of $200-8000 \mathrm{~Hz}$.

Aibara et al. [16] obtained the stapes velocity transfer function (SVTF) curve from 11 fresh temporal bones with Doppler Vibration Instrument, characterizing the middle ear sound transfer function. Stapes velocity transfer function is defined as

$$
\mathrm{SVTF}=\frac{V_{\mathrm{FP}}}{P_{\mathrm{TM}}},
$$




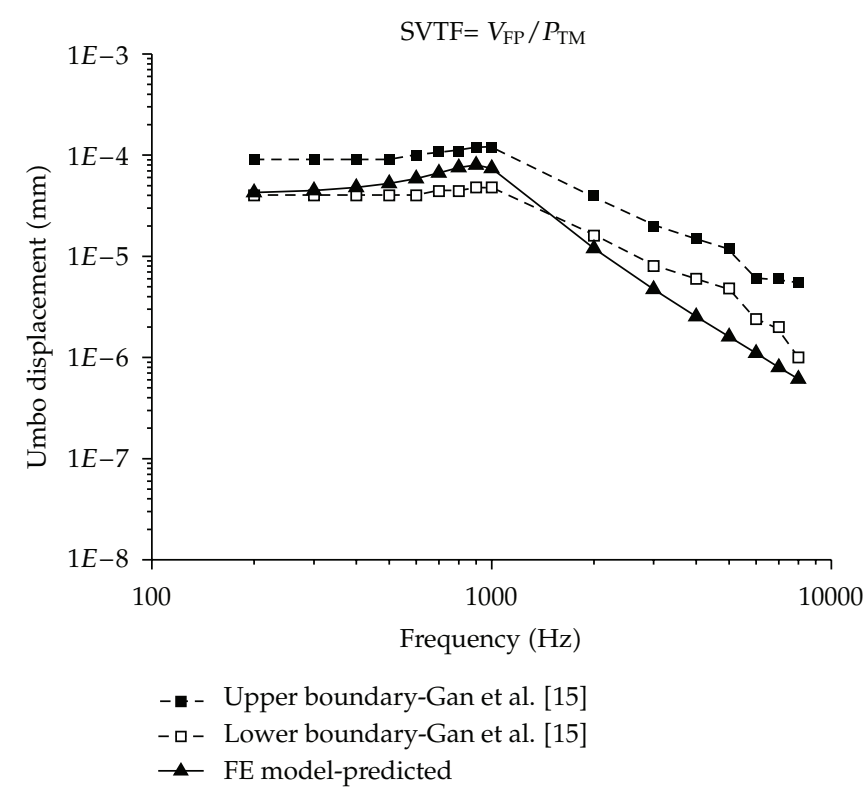

Figure 4: Comparison of the displacement of umbo between the FE model-predicted and the experimental data of Gan et al. [15].

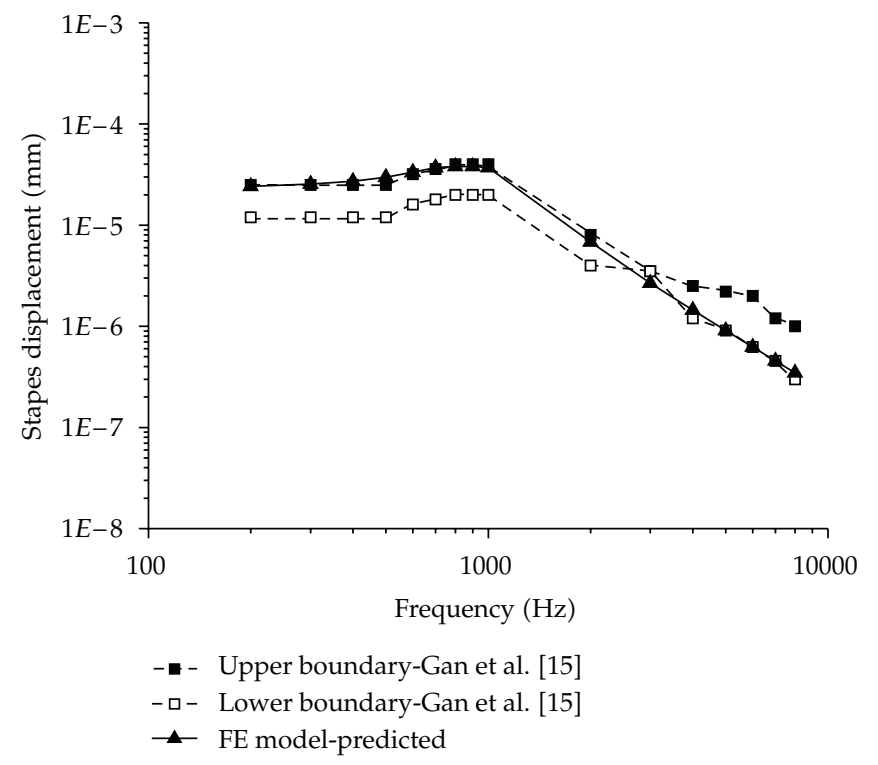

Figure 5: Comparison of the displacement of stape footplate between the FE model-predicted and the experimental data of Gan et al. [15].

where $V_{\mathrm{FP}}$ is stapes footplate speed of stapes and $P_{\mathrm{TM}}$ is pressure near the eardrum. Figure 6 shows the SVTF curve calculated by the FE model comparisons with the experimental results. 


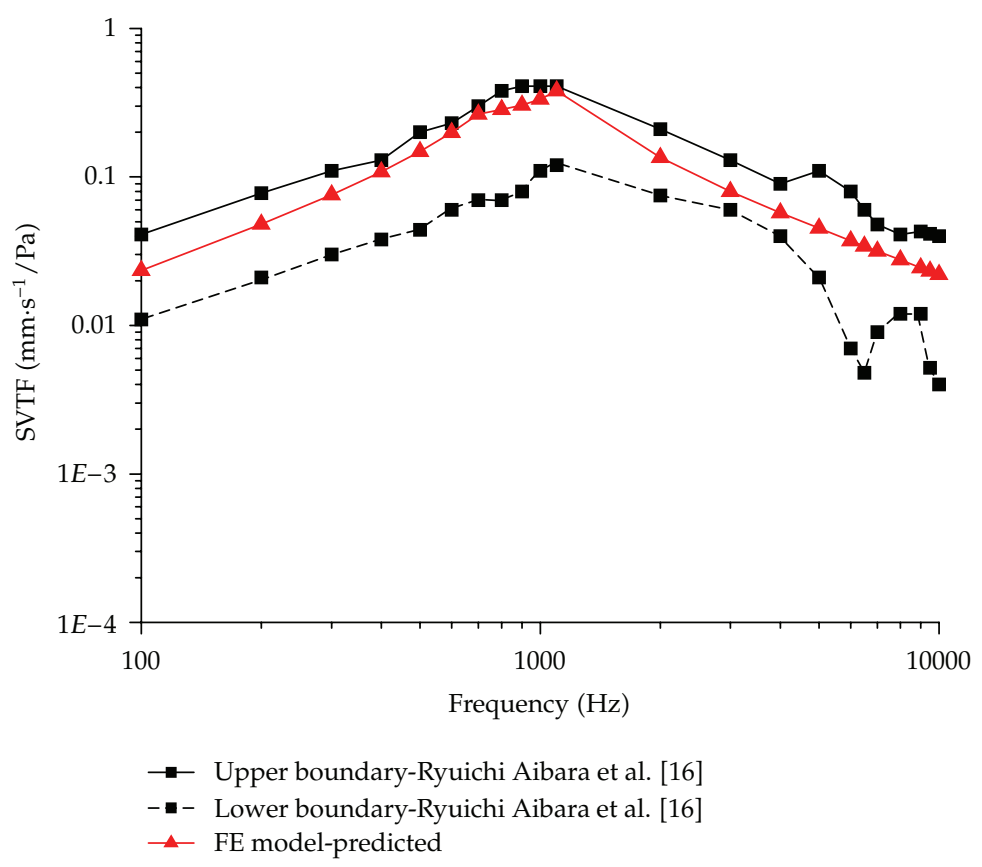

Figure 6: Comparison of the stapes footplate velocity transfer function between the FE model-predicted and the experimental data.

The displacement of soft tissue in the boundary condition and the elastic modulus of Table 1 are most sensitive and important for the results in Figures 4-6. But the displacement of tympanic membrane umbo and stapes footplate was derived from inversion.

The simulation results show that SVTF reaches the average maximum when the frequency is $1 \mathrm{KHz}$, gets $0.33 \mathrm{~mm} \mathrm{~s}^{-1} / \mathrm{Pa}$, has a slope of about $7 \mathrm{~dB}$ /octave in the range of $100-$ $1000 \mathrm{~Hz}$ frequency, and has a slope of about $-7 \mathrm{~dB}$ /octave above $1000 \mathrm{HZ}$. Figure 6 shows the SVTF calculation results by finite element model and Aibara et al. measured SVTF through the 11 cases of fresh temporal bone. The comparisons of results show that in the paper, the frequency response curves obtained by computational results and experiments are in very good agreement not only in tendency but also in amplitude, therefore, further proving that the model is correct and, thus the present model is a good starting point to predict the dynamical properties of ear structure.

\subsection{Distribution of External Ear Canal Sound Pressure}

This paper makes use of finite element model to impose sound incentive on external auditory canal, and results are compared with those of sound pressure imposed on tympanic membrane in the vicinity, the comparison shows that when the range of frequency is between 3 and $4 \mathrm{kHz}$, the effect that impose sound incentive on external auditory canal is higher than that of Eardrum with an increase of $10 \mathrm{~dB}$. The increase reaches the maximum when the frequency is $3700 \mathrm{~Hz}$; see Figure 7 . This result can be explained by the physical principle that inflatable pipe closed at one end can generate resonant interaction with the acoustic whose wavelength is 4 times tube length. The external auditory canal belongs to this type 


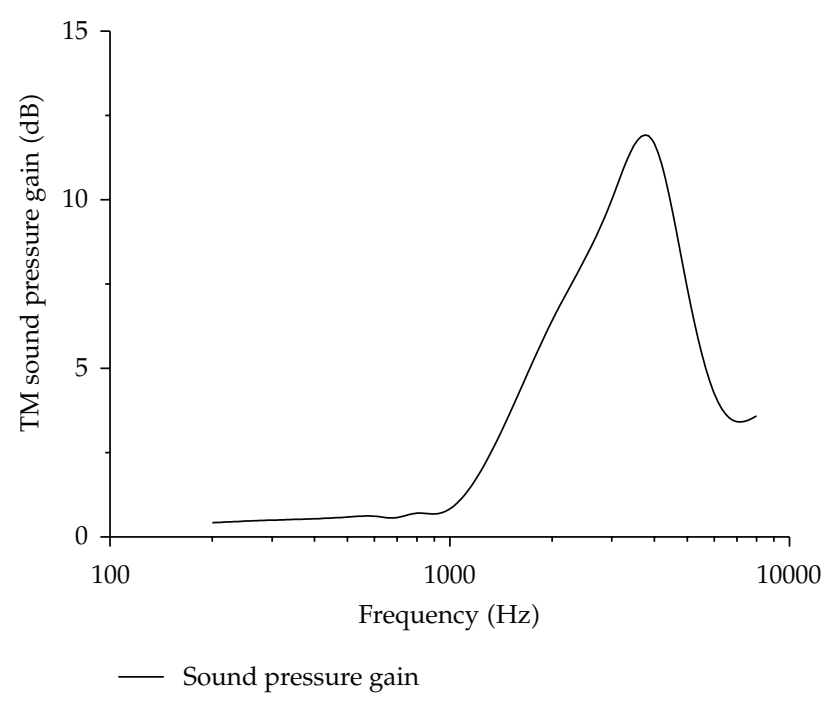

Figure 7: The pressure gain of external auditory canal for various frequencies.

tube (one end opening, the other end terminated at the tympanic membrane), and its length is about $2.5-3.5 \mathrm{~cm}$; therefore, external auditory canal plays a role of amplification in signals among the range of $3000-4000 \mathrm{~Hz}$ frequency.

Figure 8 shows the distribution of acoustic pressure in external auditory canal at different frequency; the distance of 0 is external auditory canal mouth, and the distance of $30 \mathrm{~mm}$ is convex part of eardrum. As can be seen from the figure, in the low frequency $(500 \mathrm{~Hz}, 1000 \mathrm{~Hz})$, the sound pressure in the external auditory canal at different location has no obvious difference, and the sound pressure gain is quite small. Under the frequency of $2000 \mathrm{~Hz}$, the increase of sound pressure at different location also has no obvious difference, but sound pressure has expanded, the biggest sound pressure gain is about $3 \mathrm{~dB}$. When the sound frequency is $4000 \mathrm{~Hz}$, the sound pressure gain is big, and the effect of increment varies with location: with the location being closer to the tympanic membrane, the larger of increment of sound pressure is (about $10 \mathrm{~dB})$, while the gain is quite small $(0.5 \mathrm{~dB})$ near external auditory canal mouth. At the higher frequency, there was a decrease in the sound pressure at the central location of external auditory canal. When the frequency is about $8000 \mathrm{~Hz}$, there is the largest decline at the location $17.5 \mathrm{~mm}$ away from the external auditory canal mouth $(-10 \mathrm{~dB})$, and the sound pressure still has small increase at the location near external auditory canal and tympanic membrane; the sound pressure gain is in the range of $2 \mathrm{~dB}$.

Figure 9 shows the acoustic pressure at different location of external auditory canal from $200 \mathrm{~Hz}$ to $8000 \mathrm{~Hz}$. As can be seen from the figure, in the rang of low frequency (200$1000 \mathrm{~Hz}$ ), the variation of sound pressure of external auditory canal at different location is quite small, within $1.5 \mathrm{~dB}$. Among the range of intermediate frequency $(1000 \mathrm{~Hz}-4000 \mathrm{~Hz})$, sound pressure at different position all have increased; the closer the location to the tympanic membrane, the larger the sound pressure gain, and the increasing values vary with the increment of the frequency. The increase reaches the maximum when the frequency is $4000 \mathrm{~Hz}$ (In this paper, only mapping the frequency points of integer multiple of 1000 in the high frequency, the actual maximum appears in the about $3700 \mathrm{~Hz}$.) In the higher-frequency range 


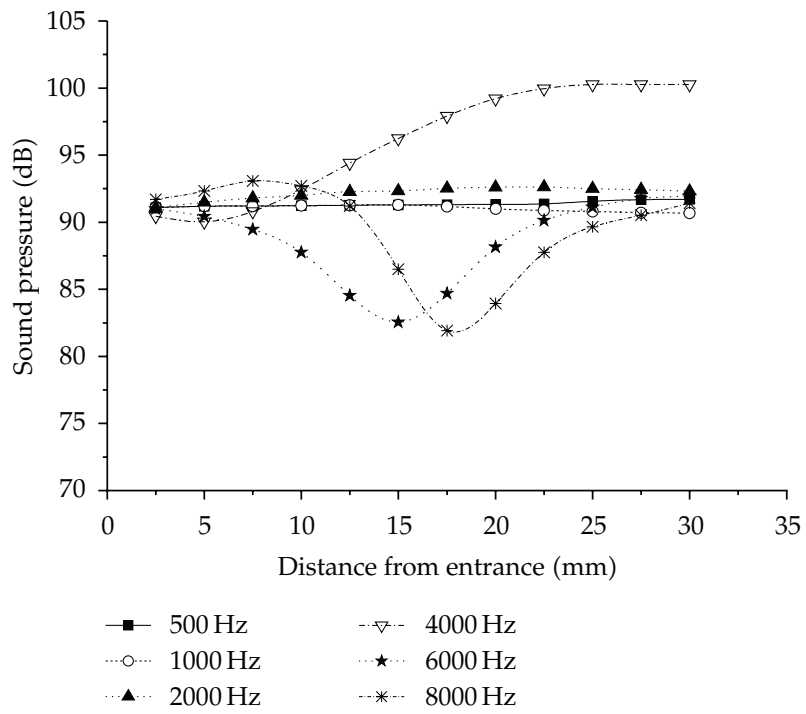

Figure 8: Distribution of sound-pressure level in the external auditory canal at frequencies of $500-8000 \mathrm{~Hz}$ $(90 \mathrm{~dB})$.

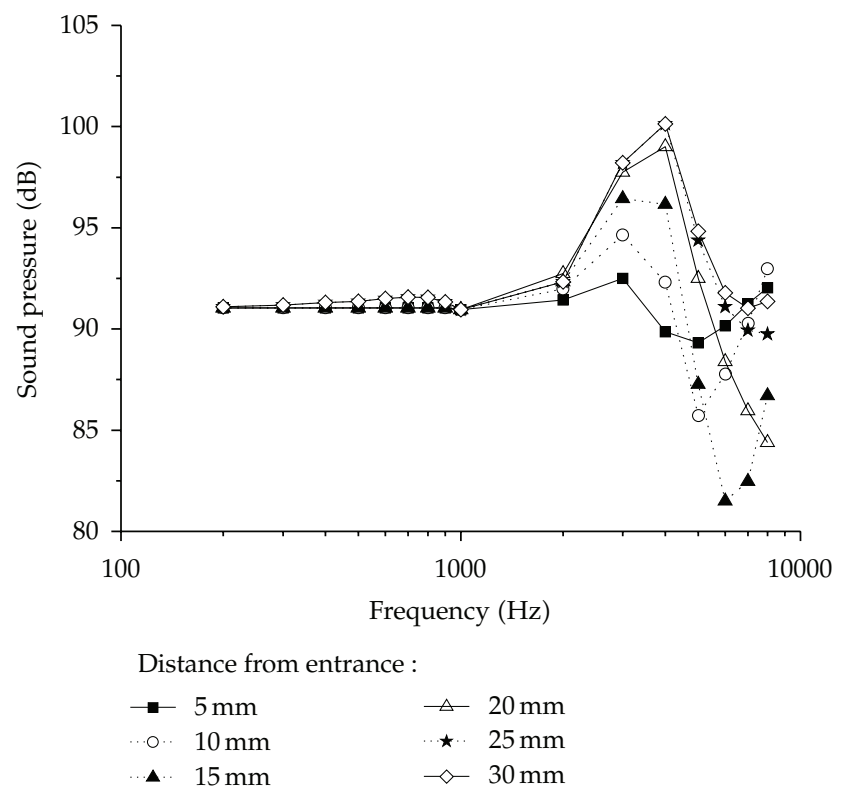

Figure 9: Frequency response curves of the sound pressure at six locations along the external auditory canal $(90 \mathrm{~dB})$.

$(4000 \mathrm{~Hz}-8000 \mathrm{~Hz})$, gain variations of sound pressure of external auditory canal at different positions are not uniform. The basic trend is declining firstly then rise slightly. But change of the magnitude is different, the reductions are all quite obvious at the location $5 \mathrm{~mm}-25 \mathrm{~mm}$ away from the external auditory canal, the increments of sound pressure are negative above the frequency of $4000 \mathrm{~Hz}$. the increments at the location $5 \mathrm{~mm}$ and $10 \mathrm{~mm}$ are positive when 


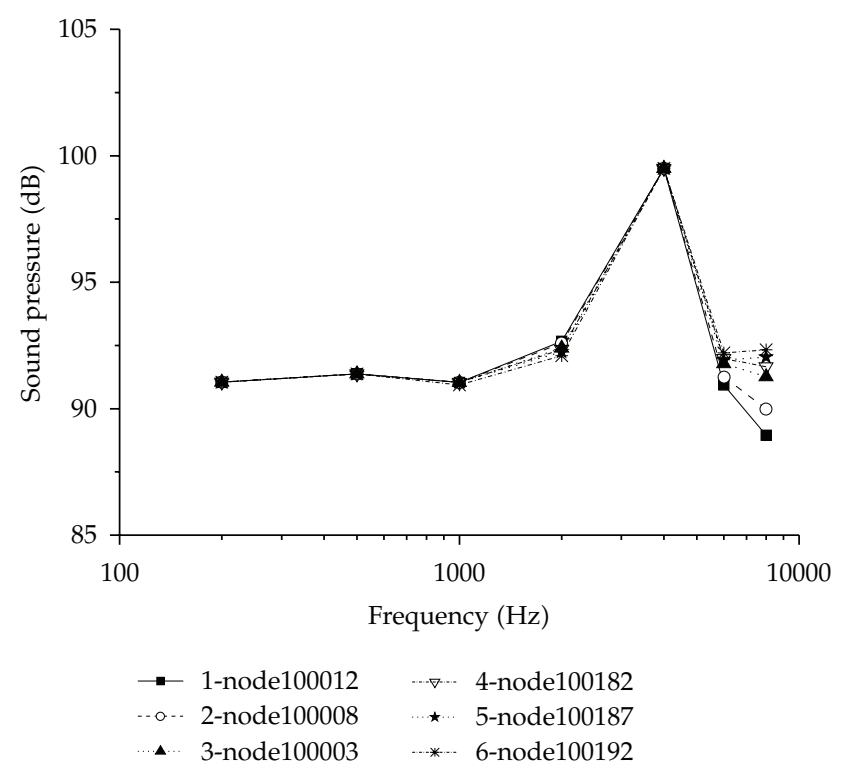

Figure 10: The variation of sound-pressure level at the eardrum for various frequencies (90 dB).

the frequency is $4000 \mathrm{~Hz}$. The declines of sound pressure near tympanic membrane are quite obvious, but the sound pressure still maintain above $90 \mathrm{~dB}$ (input sound incentive on the external acoustic foramen), the increments of sound pressure are positive.

Figure 10 shows the changing of acoustic pressure at six different locations near tympanic membrane surface varying with frequency. The figure shows that in the frequency range of $200 \mathrm{~Hz}-4500 \mathrm{~Hz}$, the distributions of different points of sound pressure are basically the same, but between the range of $4500 \mathrm{~Hz}-8000 \mathrm{~Hz}$, the difference in sound pressure appears near tympanic membrane. This phenomenon can prove that when sound pressure reaches eardrum, the tympanic membrane is equivalently incited by uniformly distributed pressure in the low-frequency range. However, when sound pressure above $4500 \mathrm{~Hz}$ frequency, different points of tympanic membrane are incited by different sound pressure, and the pressure that influences the surface of tympanic membrane is no longer uniform sound pressure.

According to calculation results, piecewise function of sound pressure which varies with frequency in different points of the surface of tympanic membrane was fitted using least-square method

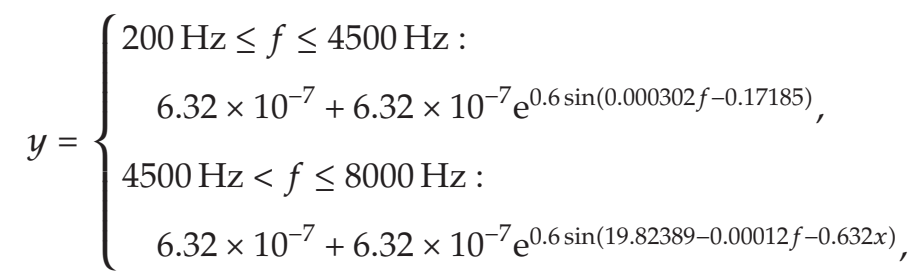

where $y$ is sound pressure (MPa), $f$ is frequency $(\mathrm{Hz})$, and $x$ is distance between different points and the umbo $(\mathrm{mm})$. 


\section{Conclusion}

The paper achieves the following conclusions by numerical simulation and theoretical analysis.

(1) The finite element model containing external auditory canal, middle ear, and inner ear hearing system was established, made use of this model to do the frequency response analysis containing gas external auditory canal, middle ear structure, and inner ear fluid coupling, and get the response curves of tympanic membrane and stapes footplate. In the paper, the curves obtained by calculation results using this model and experiment data of was in very good agreement, and prove that the model is correct.

(2) In consideration of the sound transmission role of external auditory canal, middle ear structures occurs resonance at the frequency $3000 \mathrm{~Hz}-4000 \mathrm{~Hz}$, and close to the conclusions of medical science [17], further proving that the correctness of the model.

(3) The calculation results showed that in the low frequency $(<4500 \mathrm{~Hz})$, the sound pressure that transmits uniform sound pressure of external acoustic foramen into the surface of tympanic membrane by external auditory canal mainly varies with frequency, the effects of changes in distance can be ignored, in the high frequency $(>4500)$, the situation is different; the sound pressure of the surface of tympanic membrane does not only vary with frequency, but also relates to distance. Thus, according to simulation results, functional formula of sound pressure of the surface of tympanic membrane was fitted using least-square (3.2).

(4) Previous studies usually defined a constant sound pressure in simplified model of tympanic membrane, taking no consideration of the influence of external auditory canal. Since (3.2) provide a new distribution function of sound pressure, it can be used to calculate the sound pressure at any point and further provide more accurate boundary conditions for the middle ear structure. It could provide additional information and insight for researchers to better understand the mechanism of sound transmission in human ear.

\section{Acknowledgments}

The authors gratefully acknowledge national natural science foundation of China (11072143) and foundation research key project of shanghai science committee (08jc1404700).

\section{References}

[1] Y. Wenjuan, L. H. Xingsheng, and L. Xiaoqing, "The vibration equation's establishment and solution of the eardrum," Journal of Vibration and Shock, vol. 27, no. 3, pp. 63-66, 2008.

[2] Y. Wenjuan, W. Li, and X. Li, "Analytical method for testing mechanical properties of artificial ossicular," Journal of Theoretical and Applied Mechanics, vol. 41, no. 2, pp. 216-221, 2009.

[3] S. E. Voss and W. T. Peake, "Non-ossicular signal transmission in human middle ears: experimental assessment of the "acoustic route" with perforated tympanic membranes," Journal of the Acoustical Society of America, vol. 122, no. 4, pp. 2135-2153, 2007.

[4] M. Bance, A. Campos, L. Wong, D. Morris, and R. van Wijhe, "How does prosthesis head size affect vibration transmission in ossiculoplasty?" Journal of Otolaryngology, vol. 137, no. 1, pp. 70-73, 2007. 
[5] C. Dai, M. W. Wood, and R. Z. Gan, "Combined effect of fluid and pressure on middle ear function," Journal of Hearing Research, vol. 236, no. 1-2, pp. 22-32, 2008.

[6] J. P. Vard and D. J. Kelly, "The influence of ventilation tube design on the magnitude of stress imposed at the implant/tympanic membrane interface," Journal of Medical Engineering \& Physics, vol. 30, no. 2, pp. 154-163, 2008.

[7] R. A. Tange and W. Grolman, "An analysis of the air-bone gap closure obtained by a crimping and a non-crimping titanium stapes prosthesis in otosclerosis," Journal of Auris Nasus Larynx, vol. 35, no. 2, pp. 181-184, 2008.

[8] J. Tenney, M. A. Arriaga, D. A. Chen, and R. Arriaga, "Enhanced hearing in heat-activated-crimping prosthesis stapedectomye," Journal of Otolaryngology, vol. 138, no. 4, pp. 513-517, 2008.

[9] R. Z. Gan, T. Cheng, C. Dai, and F. Yang, "Finite element modeling of sound transmission with perforations of tympanic membrane," Journal of the Acoustical Society of America, vol. 126, no. 1, pp. 243-253, 2009.

[10] R. Z. Gan, B. P. Reeves, and X. Wang, "Modeling of sound transmission from ear canal to cochlea," Annals of Biomedical Engineering, vol. 35, no. 12, pp. 2180-2195, 2007.

[11] R. Z. Gan, Q. Sun, B. Feng, and M. W. Wood, "Acoustic-structural coupled finite element analysis for sound transmission in human ear-pressure distributions," Journal of Medical Engineering and Physics, vol. 28, no. 5, pp. 395-404, 2006.

[12] Y. Wenjuan and X. L. Li, "Research on pathological changes of middle ear and artificial stapes," Journal of Medical Biomechanics, vol. 24, no. 2, pp. 118-122, 2009.

[13] Y. Wen-juan, W. Li, L. J. Fu, and X. S. Huang, "Numerical simulation and transmitting vibration analysis for middle-ear structure," Journal of System Simulation, vol. 21, no. 3, pp. 651-654, 2009.

[14] Y. Wenjuan, X. S. Huang, and L. J. Fu, "Transmitting vibration of artificial ossicle," International Journal of Nonlinear Sciences and Numerical Simulation, vol. 9, no. 2, pp. 131-139, 2008.

[15] R. Z. Gan, M. W. Wood, and K. J. Dormer, "Human middle ear transfer function measured by double laser interferometry system," Journal of Otology and Neurotology, vol. 25, no. 4, pp. 423-435, 2004.

[16] R. Aibara, J. T. Welsh, S. Puria, and R. L. Goode, "Human middle-ear sound transfer function and cochlear input impedance," Journal of Hearing Research, vol. 152, no. 1-2, pp. 100-109, 2001.

[17] X. P. LI and R. H. Zheng, Ear Anatomy and Clinic, Peking University Medical Press, Beijing, China, 2007. 


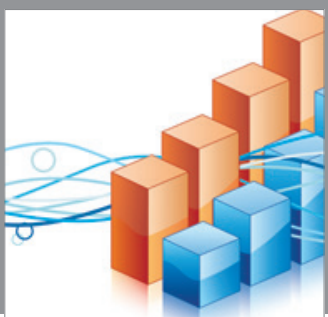

Advances in

Operations Research

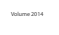

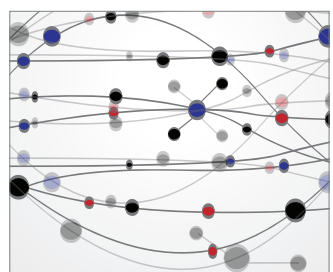

\section{The Scientific} World Journal
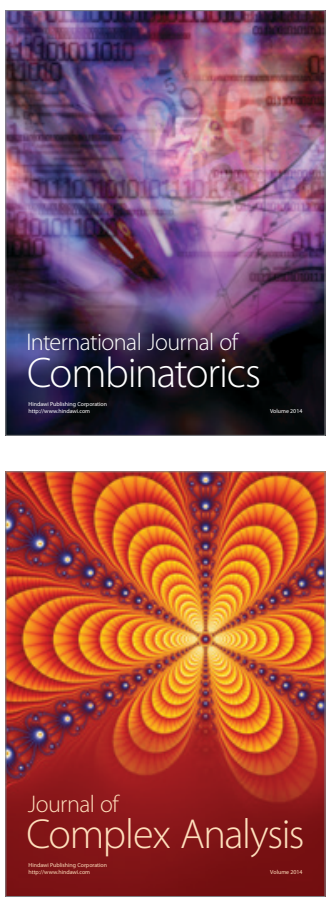

International Journal of

Mathematics and

Mathematical

Sciences
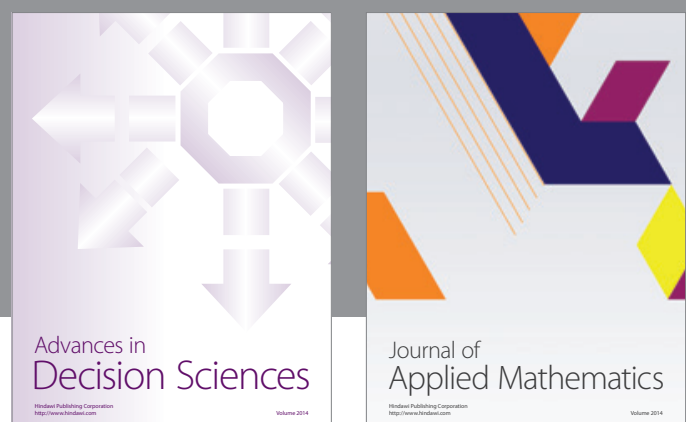

Journal of

Applied Mathematics
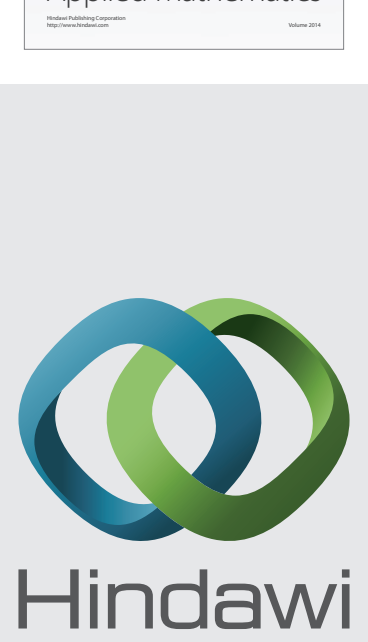

Submit your manuscripts at http://www.hindawi.com
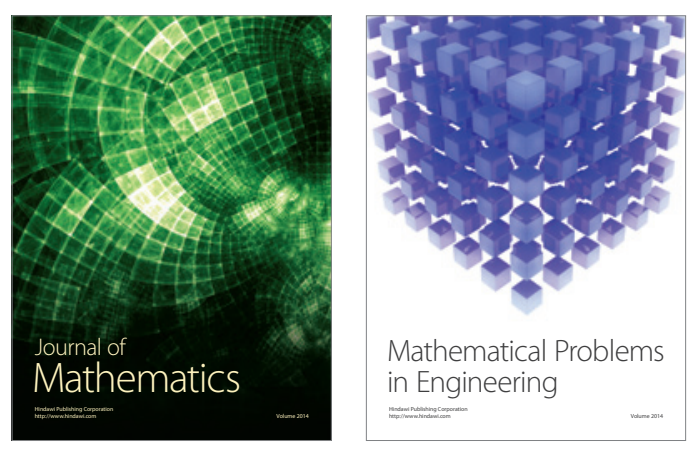

Mathematical Problems in Engineering
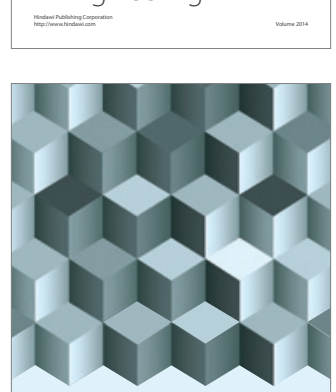

Journal of

Function Spaces
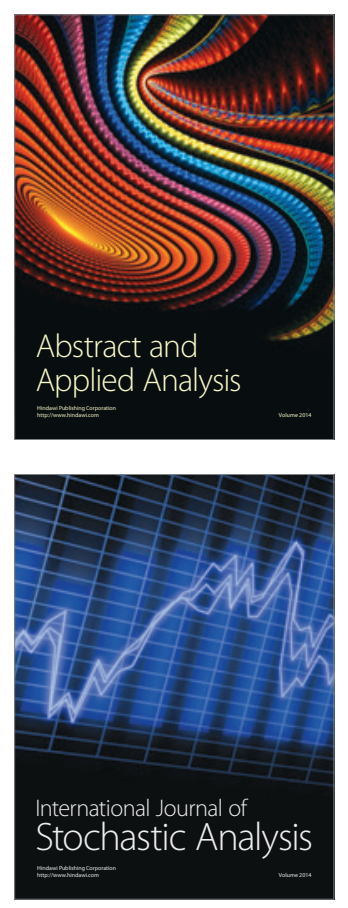

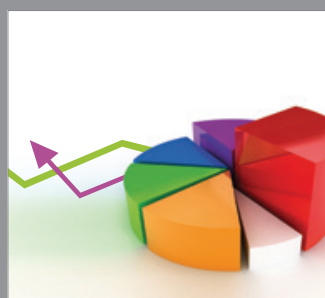

ournal of

Probability and Statistics

Promensencen
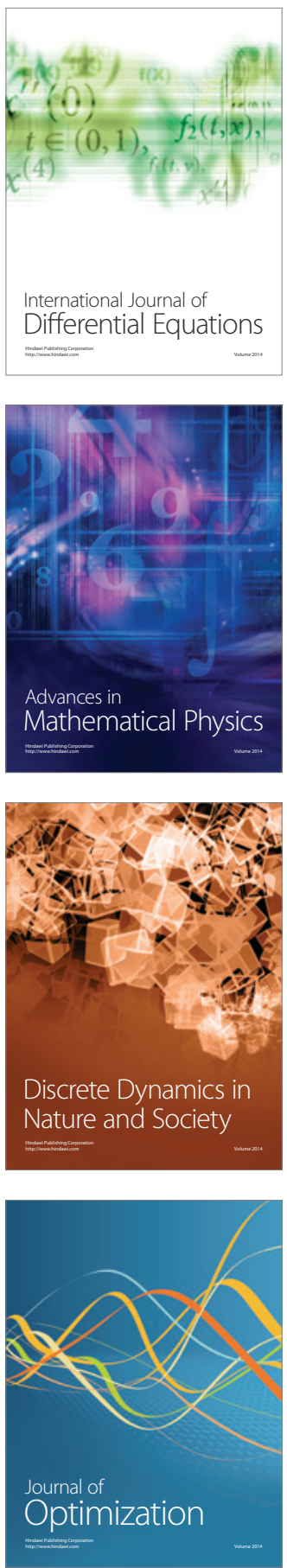